\title{
When Causality Shapes the Experience of Time: \\ Evidence for Temporal Binding in Young Children.
}

\author{
Running Title: Temporal Binding in Children \\ Emma Blakey ${ }^{1 *}$, Emma C Tecwyn ${ }^{2 *}$, Teresa McCormack $^{3}$, David A Lagnado ${ }^{4}$, Christoph \\ Hoerl $^{5}$, Sara Lorimer ${ }^{3} \&$ Marc J Buehner ${ }^{2 \dagger}$ \\ 1 The University of Sheffield, Department of Psychology \\ 2 Cardiff University, School of Psychology \\ 3 Queen's University Belfast, School of Psychology \\ 4 University College London, Department of Psychology \\ 5 University of Warwick, Department of Philosophy
}

*The first and second authors contributed equally; author order is alphabetical

${ }^{\dagger}$ Corresponding author: School of Psychology, Cardiff University, Cardiff CF10 3AT;

BuehnerM@cardiff.ac.uk; +44 (0) 2920870035

The authors have no conflict of interest to declare.

This article has been accepted for publication and undergone full peer review but has not been through the copyediting, typesetting, pagination and proofreading process, which may lead to differences between this version and the Version of Record. Please cite this article as doi: 10.1111/desc.12769

This article is protected by copyright. All rights reserved. 


\title{
Research Highlights:
}

- We demonstrate for the first time that children as young as four years are susceptible to temporal binding.

- Temporal binding in children appears to be grounded in causal knowledge rather than intentional action.

- The early-developing bidirectional relation between time and causation may be a useful heuristic that helps young children learn causal relationships.

\begin{abstract}
It is well-established that the temporal proximity of two events is a fundamental cue to causality. Recent research with adults has shown that this relation is bidirectional: events that are believed to be causally related are perceived as occurring closer together in time- the socalled temporal binding effect. Here we examined the developmental origins of temporal binding. Participants predicted when an event that was either caused by a button press, or preceded by a non-causal signal, would occur. We demonstrate for the first time that children as young as four years are susceptible to temporal binding. Binding occurred both when the button press was executed via intentional action, and when a machine caused it. These results suggest binding is a fundamental, early developing property of perception and grounded in causal knowledge.
\end{abstract}

Keywords: Causal binding, Causality, Intentional action, Temporal binding, Temporal contiguity, Time perception

This article is protected by copyright. All rights reserved. 


\section{Introduction}

Causal beliefs ground our understanding of the world: they allow us to explain our observations and make predictions. Hume (1888) made temporal contiguity - the closeness of two events in time - part of his definition of causation, and psychologists showed that this is indeed a fundamental cue in causal inference (Dickinson, 2001; Shanks, Pearson \& Dickinson, 1989; Buehner \& May, 2002, 2003). However, recent research suggests that the relation between time and causation is more complex than the Humean view suggests. Not only does the timing of events influence causal beliefs, but causal beliefs themselves also affect our perception of the timing of events. Thus, events that we believe are causally related are perceived as occurring closer together in time (Buehner, 2012; Buehner \& Humphreys, 2009; Haggard, Clark, \& Kalogeras, 2002; Moore \& Obhi, 2012).

This temporal binding effect is robust and has been demonstrated in adults using a variety of paradigms (e.g., Desantis, Hughes \& Waszak, 2012; Engbert \& Wohlschläger, 2007; Nolden, Haering \& Kiesel, 2012). It is seen as a generalized consequence of causal beliefs, rather than as occurring only in the context of intentional actions: temporal binding occurs for voluntary and involuntary (Dogge, Schaap, Custers, Wegner, Aarts, 2012; Buehner, 2015) as well as mechanical causal actions (Buehner, 2012), and is moderated by the contingency between events (Moore, Lagnado, Deal, \& Haggard, 2009).

The phenomenon of temporal binding sheds new light on the relation between time and causation in adults, but we know very little about its developmental origins. Is children's experience of time similarly influenced by their causal knowledge? Young children's causal beliefs are strongly affected by temporal information, including temporal order (Bullock \& Gelman, 1979; Rankin \& McCormack, 2013; Rottman, Kominsky \& Keil, 2013) and temporal contiguity (Mendelson \& Schultz, 1976; Schlottmann, 1999). Perhaps the 
bidirectional relationship between time and causality is a fundamental invariant property of our perceptual and cognitive system, in which case we would expect binding to be present from early childhood. There appears to be a privileged relation between time and causation in children: children prioritize temporal information over other types of cues such as statistical information (McCormack, Frosch, Patrick, \& Lagnado, 2015) and knowledge of causal mechanisms (Schlottmann, 1999), suggesting that the use of temporal information to infer causality may be automatic and developmentally basic (Schlottmann, Allen, Linderoth, \& Hesketh, 2002). It is thus possible that the close relation between time and causation seen in childhood works both ways: that children not only rely heavily on temporal cues in forming causal beliefs, but that such beliefs also affect their perception of time.

Despite the intuitive plausibility of the claim that children are susceptible to binding, the only two developmental studies that have investigated it seem to provide evidence against it. Cavazzana et al. $(2014,2017)$ found that 8 - to 11 -year-olds did not show temporal binding. This suggests a very different hypothesis: that binding emerges late. Perhaps temporal binding requires sustained experience of interacting with causal events, or perhaps this sort of top-down process requires late-developing cognitive resources. To decide between these hypotheses, we designed a new task that was much simpler than that used by Cavazzana et al., and thus could be used even with young children.

\section{Experiment 1}

Participants completed a stimulus anticipation task adapted from previous research with adults (Buehner, 2012). There were two conditions, causal and non-causal, which were each run with two target intervals (900 and 1300 ms, to give the impression of different tasks; Figure S1a). Children anticipated when a rocket or a jack-in-a-box would launch by trying to launch a matching stimulus at the same time (see Figures 1 and 2). In the causal condition, 
launching occurred at a fixed delay after participants' button press; in the non-causal condition launching occurred after an arbitrary warning signal. Thus, while the interval between the initial event (button press or warning signal) and the stimulus launch was identical in both conditions, the events were causally linked in the causal condition only. If temporal binding is present, participants should underestimate the interval between the two events in the causal condition relative to the non-causal condition, reflecting their subjective impression that the stimulus launch occurred earlier when it was caused than when the two events were not causally related.

\section{Method}

\section{Participants}

One-hundred and seventy-two children, recruited from schools $(N=92)$ and a science museum $(N=80)$ participated. We split these into four age groups: 36 four- to five-year-olds ( $M_{\text {age }}: 4.53$ years; 21 females), 55 six- to seven-year-olds ( $M_{\text {age }}: 6.38$ years; 26 females), 46 eight- to nine-year-olds ( $M_{\text {age }}: 8.65$ years; 25 females $)$, and 34 ten- to eleven-year-olds $\left(M_{\text {age }}\right.$ : 10.56 years; 19 females). An additional eleven children were excluded due to poor concentration $(N=4)$, because they did not understand the task instructions $(N=3)$, or due to technical issues $(N=4)$.

\section{Materials}

The experiment was conducted on a laptop with a screen refresh rate of $60 \mathrm{~Hz}$ running E-Prime 2.0 (Psychology software tools, Pittsburgh, PA). Participants responded using two buttons (green and yellow on the left/right) on a response box. The initial stimuli were an onscreen depiction of a jack-in-a-box (JIAB) and a rocket standing in a gantry; the target stimuli were the jack popped from its box and the rocket launched from the gantry (see Figures 1 and 2). In addition, there were matching stimuli on the right of the screen, used to elicit 
participants' anticipation of the target outcome. These were mirrored versions of the jack or rocket in the initial training phase, but were obscured by an on-screen curtain or clouds in the experimental phase. In the causal condition, the target stimulus had a depiction of a green button attached to it. This illuminated when the participant pressed the green button on the response box and was accompanied by a $200 \mathrm{~ms}$ tone. In the non-causal condition, the target had a blue light attached to the centre of the box or gantry which illuminated when the warning cue was initiated along with a $200 \mathrm{~ms}$ tone. When the target stimulus launched this was also accompanied by a $200 \mathrm{~ms}$ sound. The color of the stimuli changed when the time interval changed (from $2000 \mathrm{~ms}$ in the practice phase to $900 \mathrm{~ms}$ to $1300 \mathrm{~ms}$ in the experimental phases; see below) to highlight that these were different versions of the stimulus and so could launch at different times.

\section{Design \& Procedure}

Participants' task was to launch an identical stimulus at exactly the same time as the target stimulus. Two factors were manipulated: 1) whether the target's launch was preceded by a causal action (button press) or a non-causal signal; and 2) the delay between the causal action/non-causal signal and the target stimulus' launch $(900 \mathrm{~ms}$ or $1300 \mathrm{~ms}$, following previous work with adults, e.g. Buehner, 2012). Participants completed four 20 trial experimental blocks: one causal and one non-causal for each of the two delays (Figure S1a).

On causal trials, participants had to press the green button to produce the target launch; on non-causal trials, participants had to monitor the screen for the warning cue preceding the target launch. In causal trials, participants pressed the green button when they were ready; in non-causal trials, the warning cue occurred at a variable interval between $2300 \mathrm{~ms}$ and 2800ms after trial intialization. On causal trials, participants' button press immediately triggered a 200ms beep; in addition, the on-screen representation of a green button illuminated for $200 \mathrm{~ms}$. On non-causal trials, the warning cue consisted of a $200 \mathrm{~ms}$ beep and 
an on-screen illumination of a blue light mounted on either the rocket gantry or the JIAB for 200ms. This was done to keep the audio-visual experience of causal and non-causal trials as similar as possible. Following a set interval of $900 \mathrm{~ms}$ or $1300 \mathrm{~ms}$ after the causal action or non-causal signal, the target stimulus launched accompanied by a 200ms sound (either a rocket launch sound or a "boing" sound). Participants' task was to anticipate the target's appearance by pressing the yellow button on the response box to launch a matching stimulus so that it appeared at exactly the same time. The matching stimulus always appeared immediately upon pressing the yellow button. The dependent variable was participant's judgment error - the time they launched the matching stimulus relative to the actual appearance of the target stimulus.

Each condition had a demonstration phase (4 trials), a practice phase (to criterion of 3 trials correct), and two experimental phases (20 trials for each time interval; Figure S1a). In the demonstration phase, the matching stimulus appeared on its own and participants launched it four times using the yellow button, demonstrating that the matching stimulus launched immediately upon button press. In the practice phase, participants were introduced to the target stimulus, and they practiced launching the matching stimulus at the same time as the target stimulus. In this phase, the target stimulus launched after 2000ms (so the intervals experienced in the test trials were novel) after the participants' first button press (causal trials) or the warning cue (non-causal trials). Participants were told that their task was to ensure that the faster matching stimulus launched at the same time as the slower target stimulus; there was a cover story according to which the jacks wanted to jump out at the same time/the astronauts in the rockets wanted to launch at the same time because they were friends. In the causal condition they were then told: "You press the green button here to make this jack-in-a-box/rocket start to get ready to pop, wait just a little while, and then you press the yellow button here at exactly the time you think the first jack/rocket will appear. Try not 
to press the yellow button too early or too late, you want both jacks/rockets to pop out at the same time. If you manage to make them pop out at the same time, you will get a point and a smiley face will appear on the screen." Instructions for the non-causal condition were the same but participants were told that a light would come on and a sound play (the warning signal) when the jack/rocket on the left-hand side of the screen was getting ready to launch. Participants had to get three trials in a row correct (defined as launching the matching stimulus within a $1000 \mathrm{~ms}$ window centred on the target launch time) to proceed to the experimental phase. A smiley face appeared each time they got a correct response; this feedback was only provided during practice.

In the experimental phase, participants were told that there were different colored rockets/jacks in each block, and that these took slightly different amounts of time to launch. Also, the matching stimulus was covered up with either an on-screen curtain (for the JIAB) or a blue cloud (for the rocket). This was to ensure that participants had no visual feedback about whether their launch coincided with the target stimulus. Three factors were counterbalanced between participants: 1) condition order; 2) assignment of rocket and JIAB to the two conditions; and 3) order of intervals within each condition.

\section{Results}

Judgment errors (JEs) were calculated by subtracting the time the target occurred from the prediction response time; negative and positive scores reflect early and late prediction of the target event, respectively. Each participant contributed one median JE per condition to the analysis (see Buehner 2012 for a similar approach), calculated from their last 10 trials in a block. Response times that were $<200 \mathrm{~ms}$ or $>2000 \mathrm{~ms}$ were removed, resulting in the loss of $3.7 \%$ of trials (the number of trials excluded did not differ significantly between conditions, see Table S1 and accompanying text). Preliminary analyses of JEs found no 
effects of gender, condition order or stimulus assignment and no significant interactions; these factors were not analysed further.

A 4 (age group) x 2 (time interval) x 2 (causality: causal or non-causal) mixed ANOVA showed that JEs were more anticipatory in the causal $(M=-154.2 \mathrm{~ms})$ than the noncausal condition $(M=-66.8 \mathrm{~ms}), F(1,167)=31.43, p<.001, \eta_{p}^{2}=.16\left(M_{\mathrm{diff}}=87.44 ; 95 \% \mathrm{CI}\right.$

$[56.65,118.24]$, Figure 3$)$. JEs were also more anticipatory at $1300 \mathrm{~ms}(M=-156.0 \mathrm{~ms})$ than at $900 \mathrm{~ms}(M=-64.9 \mathrm{~ms}), F(1,167)=58.29, p<.001, \eta_{p}^{2}=.26\left(M_{\mathrm{diff}}=91.08 ; 95 \% \mathrm{CI}[67.53\right.$, 114.64]). There was also a main effect of age group $\left(F(3,167)=5.12, p=.002, \eta_{p}^{2}=.08\right)$, with 4- to 5-year-olds showing more anticipatory responses than all other age groups (posthoc pairwise comparisons, $p s<0.005$ ). These main effects were qualified by an interval by age group interaction, $\left(F(3,167)=11.07, p<.001, \eta_{p}^{2}=.17\right)$. Simple effect analyses revealed that JEs were significantly more anticipatory at $1300 \mathrm{~ms}$ than $900 \mathrm{~ms}$ for the three youngest age groups $(p s<.017)$ but not 10 - to 11-year-olds $(p=.147)$.

To examine whether binding magnitude changed developmentally, we calculated binding scores by subtracting participants' JEs in the causal from JEs in the non-causal condition for each interval (Figure 4). A 4 (age group) x 2 (interval) mixed ANOVA revealed no significant effects of age group $\left(F(3,167)=1.60, p=.192, \eta^{2}=.03\right)$ or interval $(\mathrm{F}(1$, $\left.167)=0.70, p=.792, \eta^{2}=.00\right)$ on binding score, and no interaction $(p=.937)$.

\section{Discussion}

The results suggest that causal beliefs do shape children's experience of time: 4- to 11-year-olds predicted an event would occur earlier when it resulted from a prior causal action (button press) compared to when it merely followed a predictive signal. This is the first evidence that temporal binding is present in children as young as four years. Our findings 
contrast with those of Cavazzana et al. $(2014,2017)$ in suggesting that the relation between time and causality is bidirectional early in development.

While we aimed to make the conditions of Experiment 1 as similar as possible in terms of surface-level features, the fact that participants executed the initial button press in the causal condition introduced some potential confounds. First, the two conditions differed with respect to the attentional and motor demands: specifically, the causal condition required participants to plan and execute two motor actions rather than one, and the fact that only the causal trials were self-initiated could have meant that children were more attentive in this condition. In addition, the results of Experiment 1 do not allow us to establish whether temporal binding in children is driven by the causal relation between events (as has been in adults, Buehner, 2012; Buehner \& Humpreys, 2009), or whether intentional action is critical, as these factors were confounded in the causal condition. It is also possible that causality is sufficient for binding to occur in children but intentionality modulates the effect: there is some evidence that binding in adults is stronger when the cause is one's own intentional action (Buehner, 2012) although this has not been consistently demonstrated (Poonian, McFadyen, Ogden, \& Cunnington, 2015). There is also evidence to suggest that children's actions have a special status in causal learning (Kushnir \& Gopnik, 2005; Meltzoff, Waismeyer \& Gopnik, 2012), though there are also cases that suggest no advantage for learning on the basis of one's own actions (McCormack et al., 2016). Thus, while we predict that causality will be sufficient for binding to occur in children, it is unclear whether temporal binding is expected to be stronger in children when the cause is their own action.

Experiment 2 aimed to (1) replicate the binding effect in children; (2) rule out that differences in attention and motor demands might explain our Experiment 1 results; and (3) tease apart the relative contributions of causality and intentional action to the temporal binding effect.

This article is protected by copyright. All rights reserved. 


\section{Experiment 2}

We used the same task, but introduced a third machine-causal condition, where a nonintentional machine pressed the button to launch the stimulus. Thus, there were three conditions (non-causal, self-causal and machine-causal), each run with a single target interval of $900 \mathrm{~ms}$ (Figure S1b). If attention and motor demands drove what looked like a binding effect in Experiment 1, then in Experiment 2 JEs should be comparable in the noncausal and machine-causal conditions, given that both conditions only involve performing a single action and neither of these trial-types is self-initiated. With regards to disentangling the contributions of causality and intentionality: if binding results from a causal connection between action and outcome, it should persist when the initiating action (button press) is not intentional in nature, and this would be reflected by more anticipatory JEs in the machinecausal and self-causal conditions than the non-causal condition. If, on the other hand, intentionality is the critical component, binding should only be seen in the self-causal condition. Finally, if causality is sufficient for binding but intentionality modulates the effect, then we should find binding in both conditions, but it should be stronger in the self-causal than in the machine-causal condition.

\section{Method}

\section{Participants}

Ninety-six children from schools $(N=77)$ and a science museum $(N=19)$ participated: 29 four- to five-year-olds ( $M_{\text {age }}: 5.00$ years; 16 females), 34 six- to seven-yearolds ( $M_{\text {age }}: 6.86$ years; 23 females) and 33 eight- to nine-year-olds ( $M_{\text {age }}: 8.99$ years; 22 females). An additional eight children were excluded due to poor concentration $(N=2)$, because they did not understand the task instructions $(N=4)$, or due to technical issues $(N=$ 2). 


\section{Materials}

The materials used were identical to those in Experiment 1, with the following exceptions. The stimuli consisted only of different colored rockets and the interval in the experimental phases was always 900ms. A custom-built machine with a timer, red button, and protruding lever (see Buehner, 2012, for details) was used in the machine-causal condition.

\section{Design and procedure}

The design and procedure were similar to that used in Experiment 1, with the following exceptions. As the interval was always $900 \mathrm{~ms}$, the only factor manipulated was the event that preceded the rocket launch. There was an additional machine-causal condition, in which the button was pressed by a machine that was physically separate from the rest of the apparatus. Participants completed three blocks of 20 trials (in a counterbalanced order): one self-causal, one machine-causal and one non-causal. At the start of the machine-causal block, participants were introduced to the machine. They were told that once the machine was started by the experimenter pressing the red button, this started the timer running, and every $2-5$ s the lever would drop down. This was demonstrated to the participant before aligning the machine with the response box. During the machine-causal trials, the machine was positioned adjacent to the left-hand-side of the response box, and every $2-5 \mathrm{~s}$ the lever dropped down, which pressed the green button to produce the target launch. As in Buehner (2012) the lever was actually triggered via the experimenter surreptitiously pressing a remote hidden in her pocket. In all conditions, participants had to anticipate the target's appearance by pressing the yellow button to launch the matching stimulus at exactly the same time.

This article is protected by copyright. All rights reserved. 


\section{Results}

$2.8 \%$ of trials were removed as outliers from the last 10 trials of the block. The number of trials excluded did not differ significantly between conditions (see Table S1 and accompanying text). Preliminary analyses showed that there were no significant effects of gender or condition order on JEs. There was however a significant interaction between condition order and condition $\left(F(4,156)=5.0, p=.001, \eta_{p}^{2}=.114\right.$; Figure S2), so condition order was included in subsequent analyses.

A 3 (age group) x 2 (causality: self-causal, machine-causal, or non-causal) x 3 (condition order) mixed ANOVA revealed a significant effect of causality on participants' JEs $\left(F(2,174)=10.00, p<.001, \eta_{p}^{2}=.10\right)$. Participants were most anticipatory in the selfcausal condition $(M=-69.52 \mathrm{~ms})$, least anticipatory in the non-causal condition $(M=$ $11.49 \mathrm{~ms})$, with JEs for the machine-causal condition falling in between $(M=-43.45 \mathrm{~ms}$; Figure 5).

Posthoc pairwise comparisons revealed a significant difference between JEs in the self-causal and non-causal conditions $\left(p<.001 ; M_{\text {diff }}=81.01 ; 95 \%\right.$ CI $\left.[47.35,114.67]\right)$, as well as between the machine-causal and non-causal conditions $\left(p=.006 ; M_{\text {diff }}=54.94 ; 95 \%\right.$ CI $[16.10,93.78]$ ). JEs did not differ significantly between the two causal conditions (selfcausal and machine-causal; $p=0.171)$. The ANOVA also revealed a significant main effect of age group $\left(F(1,87)=9.07, p<.001, \eta_{p}^{2}=.17\right)$, with 4- to 5-year olds being significantly more anticipatory than the other age groups $(p s<.005)$. The interaction between age group and causality was not significant $(p=.706)$.

This article is protected by copyright. All rights reserved. 
To examine whether the magnitude of binding varied developmentally, we ran a 3 (age group) x 2 (binding type: self or machine) x 3 (condition order) mixed ANOVA on participants' binding scores (Figure 4b). We found no significant effect of age group $(F(1$, $\left.87)=.62, p=.539, \eta_{p}^{2}=.01\right)$ or binding type $\left(F(1,87)=1.90, p=.171, \eta_{p}^{2}=.02\right.$; Figure 4$)$.

\section{Discussion}

Experiment 2 replicated the temporal binding effect in children, as well as the lack of any developmental change in strength of binding. The results also rule out the possibility that differences in attention and motor demands alone could have driven binding in Experiment 1. If this were the case we would have expected comparable JEs for the non-causal and machine-causal conditions of Experiment 2, as both conditions required children to execute a single key press. But JEs were significantly more anticipatory in the latter condition.

The fact that children show temporal binding when the cause was a non-intentional machine supports the notion that it is a causal connection rather than intentional action that is necessary for binding to occur in children, as has been shown for adults (Buehner, 2012). This conclusion is corroborated by the fact that binding magnitude did not differ significantly if the button was pressed by the participant or by the machine. However, given that machinecausal JEs were intermediate between non-causal and self-causal JEs, further exploration of the possibility that binding in children is modulated by intentional action is warranted.

\section{General Discussion}

We examined whether children's causal beliefs shape their temporal experience. In contrast to Cavazanna et al. (2014, 2017), who argued that binding is late-developing and not present in 8- to 11-year-olds, the two experiments reported here provide evidence of temporal binding in children from four years of age. Specifically, young children predicted that a target event would occur earlier if it was causally connected to a preceding event, compared to 
when it was preceded by an arbitrary predictive signal. Furthermore, our results suggest that binding occurs both if the cause is intentional or mechanical in nature. This demonstrates that, as is the case for adults, binding for children is grounded in causal knowledge rather than intentionality.

Our findings support an emerging body of research showing that top-down causal representations exert an influence on temporal perception (Bechlivanidis \& Lagnado, 2013; 2016). The results suggest that even for young children, temporal perception involves active interpretation of bottom-up information based on available causal knowledge. Thus, time and causality appear to mutually constrain each other already early in development: prior work shows that temporal contiguity is instrumental in forming children's causal beliefs (e.g., Schlottmann, 1999), and the present study demonstrates that once such beliefs are established, events in a causal sequence are perceived as more contiguous with each other than events in a non-causal one. While there was no overall effect of age on temporal binding, inspection of the graphs across both experiments shows that binding magnitudes were generally larger in younger children and this warrants further investigation.

Speculatively, enhanced binding early in development would yield experiences in which the temporal contiguity between cause and effect is enhanced, which could help to preserve a default assumption (that events that occur close together in time are causally related), thus facilitating causal learning in young children.

In conclusion, our findings support the idea of an early-developing relation between time and causation and extend its scope by indicating that this relation is bidirectional. Thus, our study is an important step in further elucidating how time and causation are related by shedding new light on the developmental origins of this relation.

This article is protected by copyright. All rights reserved. 


\section{Acknowledgements}

We thank the children and parents for their participation in this study, and the schools and museum who kindly hosted us. This research was supported by a Leverhulme Trust Research Project Grant (RPG-2015-267).

\section{References}

Bechlivanidis, C. \& Lagnado, D. A. (2016). Time reordered: Causal perception guides the interpretation of temporal order, Cognition, 146, 58-66. https://doi.org/10.1016/j.cognition.2015.09.001

Bechlivanidis, C., \& Lagnado, D. A. (2013). Does the "why" tell us the "when"? Psychological Science, 24(8), 1563-1572. https://doi.org/10.1177/0956797613476046

Buehner, M. J., (2012). Understanding the past, predicting the future: Causation, not intentional action, is the root of temporal binding. Psychological Science, 23, 14901497. https://doi.org/10.1177/0956797612444612

Buehner, M. J. (2015). Awareness of voluntary and involuntary causal actions and their outcomes. Psychology of Consciousness: Theory, Research, and Practice, 2(3), 237252.

Buehner, M. J., \& Humphreys, G. R. (2009). Causal binding of actions to their effects. Psychological Science, 20, 1221-1228. https://doi.org/10.1111/j.14679280.2009.02435.x

Buehner, M. J., \& May, J. (2002). Knowledge mediates the timeframe of covariation assessment in human causal induction. Thinking and Reasoning, 8, 269-295. https://doi.org/10.1080/13546780244000060

This article is protected by copyright. All rights reserved. 
Buehner, M. J., \& May, J. (2003). Rethinking temporal contiguity and the judgement of causality: Effects of prior knowledge, experience, and reinforcement procedure. The Quarterly Journal of Experimental Psychology A: Human Experimental Psychology, 56, 865-890. https://doi.org/10.1080/02724980244000675

Bullock, M., \& Gelman, R. (1979). Preschool children's assumptions about cause and effect: temporal ordering. Child Development, 50,89-96. DOI: 10.2307/1129045

Cavazzana, A., Begliomini, C., \& Bisiacchi, P. S. (2014). Intentional binding effect in children: Insights from a new paradigm. Frontiers in Human Neuroscience, 8. https://doi.org/10.3389/fnhum.2014.00651

Cavazzana, A., Begliomini, C., \& Bisiacchi, P. S. (2017). Intentional binding as a marker of agency across the lifespan. Consciousness and Cognition, 52, 104-114. https://doi.org/10.1016/j.concog.2017.04.016

Desantis, A., Hughes, G., \& Waszak, F. (2012). Intentional binding is driven by the mere presence of an action and not by motor prediction. PLoS One, 7, e29557. https://doi.org/10.1371/journal.pone.0029557

Dickinson, A. (2001). Causal learning: An associative analysis. Quarterly Journal of Experimental Psychology, 49, 60-80. https://doi.org/10.1080/713932741

Dogge, M., Schaap, M., Custers, R., Wegner, D.M., Aarts, H. (2012). When moving without volition: implied self-causation enhances binding strength between involuntary actions and effects. Consciousness and Cognition, 21(1), 501-506. https://doi.org/10.1016/j.concog.2011.10.014

Engbert, K. \& Wohlschläger, A. (2007). Intentions and expectations in temporal binding. Consciousness and Cognition, 16, 25-264. https://doi.org/10.1016/j.concog.2006.09.010

This article is protected by copyright. All rights reserved. 
Haggard, P., Clark, S., \& Kalogeras, J. (2002). Voluntary action and conscious awareness. Nature Neuroscience, 5, 382-385. https://doi.org/10.1038/nn827

Hume, D. (1888). A Treatise of Human Nature. In L. A. Selby-Bigge (Ed.). Oxford, UK: Clarendon Press.

Kushnir, T., \& Gopnik, A. (2005). Young children infer causal strength from probabilities and interventions. Psychological Science, 16, 678-683. https://doi.org/10.1111/j.14679280.2005.01595.x

Meltzoff, A.N., Waismeyer, A., \& Gopnik, A. (2012). Learning about causes from people: observational causal learning in 24-month-old infants. Developmental Psychology, 48, 1215-1228. http://dx.doi.org/10.1037/a0027440

McCormack, T., Bramley, N., Frosch, C., Patrick, F., \& Lagnado, D. A. (2016). Children's use of interventions to learn causal structure. Journal of Experimental Child Psychology, 41(2), 395-416. https://doi.org/10.1016/j.jecp.2015.06.017

McCormack, T., Frosch, C., Patrick, F., \& Lagnado, D. A. (2015). Temporal and Statistical Information in Causal Structure Learning. Journal of Experimental Psychology. Learning, Memory, and Cognition, 141, 1-22. http://dx.doi.org/10.1037/a0038385

Mendelson, R. \& Schultz, T. R. (1976). Covariation and temporal contiguity as principles of causal inference in young children. Journal of Experimental Child Psychology, 22, 408-412.

Moore, J. Lagnado, D. A., Deal, D. C., \& Haggard, P. (2009). Feelings of control: Contingency determines experience of action. Cognition, 110, 279-283. https://doi.org/10.1016/j.cognition.2008.11.006

This article is protected by copyright. All rights reserved. 
Moore, J. W., \& Obhi, S. S. (2012). Intentional binding and the sense of agency: A review. Consciousness and Cognition, 21, 546-561. https://doi.org/10.1016/j.concog.2011.12.002

Nolden, S., Haering, C. \& Kiesel, A. (2012). Assessing intentional binding with the method of constant stimuli. Consciousness and Cognition, 21(3), 1176-1185. https://doi.org/10.1016/j.concog.2012.05.003

Poonian, S. K., McFadyen, J., Ogden, J., \& Cunnington, R. (2015). Implicit agency in observed actions: evidence for N1 suppression of tones caused by self-made and observed actions. Journal of Cognitive Neuroscience, 27(4), 752-764. https://doi.org/10.1162/jocn_a_00745

Rankin, M. L., \& McCormack, T. (2013). The temporal priority principle: at what age does this develop? Frontiers in Psychology, 4. https://doi.org/10.3389/fpsyg.2013.00178

Rottman, B. M., Kominsky, J. F., \& Keil, F. C. (2013). Children Use Temporal Cues to Learn Causal Directionality, Cognitive Science, 1-25. https://doi.org/10.1111/cogs.12070

Schlottmann, A. (1999). Seeing in happen and knowing how it works: how children understand the relation between perceptual causality and underlying mechanism. Developmental Psychology, 35, 303-317.

Schlottmann, A., Allen, D., Linderoth, C., \& Hesketh, S. (2002). Perceptual causality in children. Child Development, 73, 1656-1677. https://doi.org/10.1111/14678624.00497

Shanks, D. R., Pearson, S. M., \& Dickinson, A. (1989). Temporal contiguity and the judgement of causality by human subjects. The Quarterly Journal of Experimental Psychology B: Comparative and Physiological Psychology, 41, 139-159.

This article is protected by copyright. All rights reserved. 


\section{Figure Captions}

Figure 1. The stimuli used in the Causal Condition of the Stimulus Anticipation task for the rocket and JIAB versions. Each of the three segments depicts a section of the trial. The target stimulus is the one on the left in each segment; and the matching stimulus is on the right covered by either a curtain or a cloud. Note that the curtain/cloud are depicted smaller than they actually were; on screen they were large enough to cover the matching stimulus. In the causal condition, the participant presses the green button on the response box (not depicted) to initiate the launch of the target stimulus. The button on the screen lights up to show it had been pressed and a tone is sounded - both lasting 200ms. After the set interval the test stimulus appears. The task was for participants to press the yellow button to launch the matching stimulus (under the curtain or cloud) at exactly the same time.

Figure 2. The stimuli used in the Non-Causal Condition of the Stimulus Anticipation task for the rocket and JIAB. Each of the three segments depicts a section of the trial. The target stimulus is the one on the left in each segment; and the matching stimulus is on the right covered by either a curtain or a cloud. Note that the curtain/cloud are depicted smaller than they actually were; on screen they were large enough to cover the matching stimulus. In the non-causal condition, the task was the same as the causal condition, except that participants waited for the warning cue on the rocket gantry. The warning cue was a 200ms audio-visual signal. As in the causal condition, after the set interval the test stimulus appeared, and the task was for participants to press the yellow button to launch the matching stimulus at exactly the same time.

This article is protected by copyright. All rights reserved. 
Figure 3. Mean judgement error for each causal condition (causal, non-causal) and interval (top: 900ms; bottom: 1300ms) across age groups in Experiment 1 (error bars are \pm SEM).

Figure 4. The magnitude of binding (in ms) \pm SEM for each age group (a) in Experiment 1 for $900 \mathrm{~ms}$ (top) and 1300ms (bottom) trials; and (b) in Experiment 2 for the self-causal (top) and machine-causal (bottom) conditions (both at $900 \mathrm{~ms}$ ).

Figure 5. Mean judgement error for each causal condition (self-causal, machine-causal, non-causal) and age group in Experiment 2 (error bars are \pm SEM).

This article is protected by copyright. All rights reserved. 
Figure 1

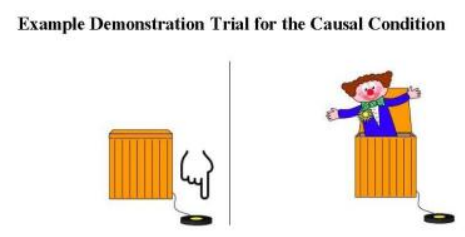

Example Practice Trial for the Causal Condition
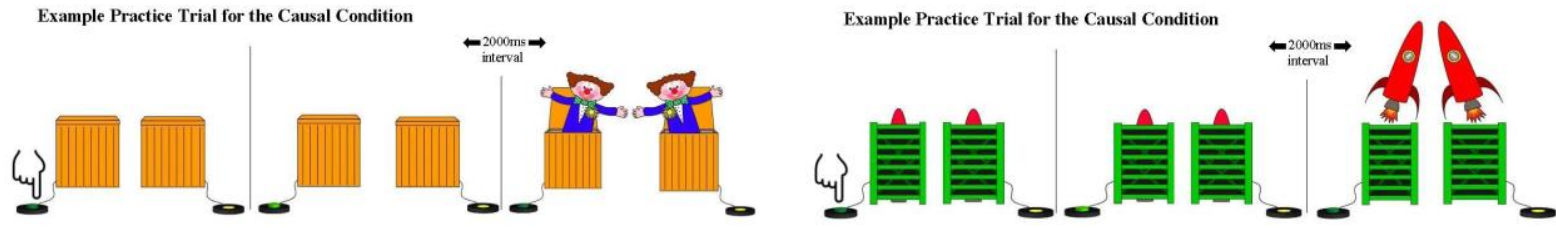

Example Experimental Trial for the Causal Condition
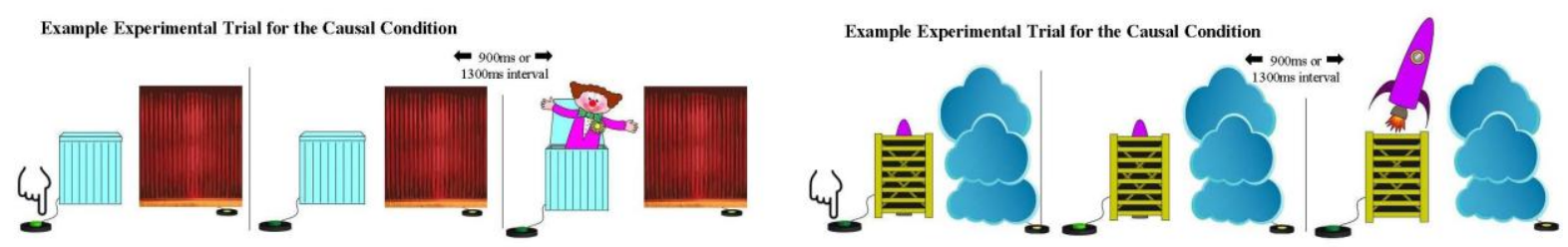

This article is protected by copyright. All rights reserved. 
Example Demonstration Trial for the Non-Causal Condition

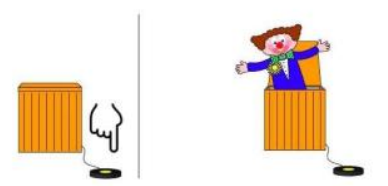

Example Practice Trial for the Non-Causal Condition

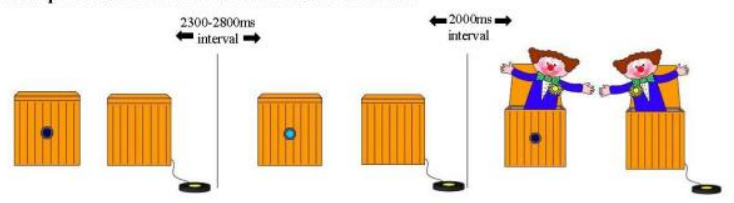

Example Experimental Trial for the Non-Causal Condition

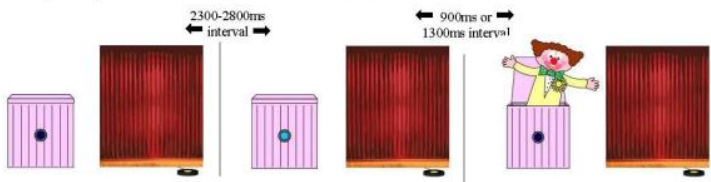

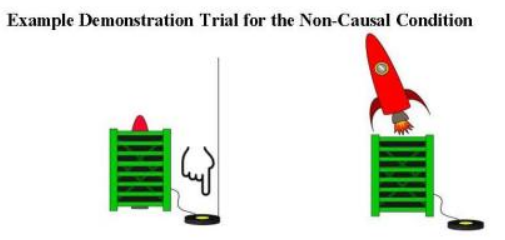

Example Practice Trial for the Non-Causal Condition

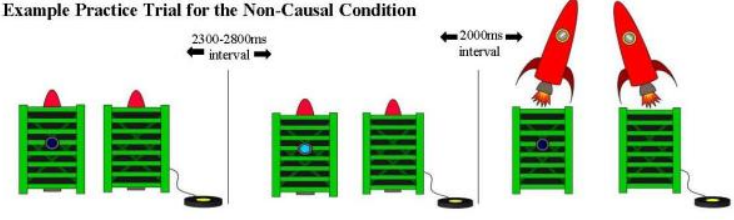

Example Experimental Trial for the Non-Causal Condition

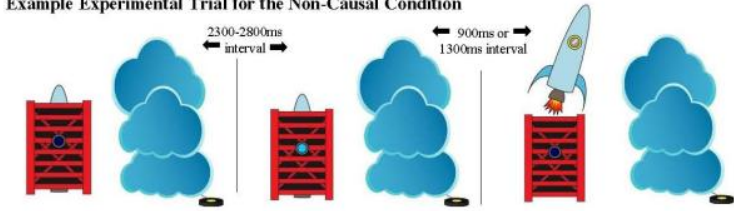

This article is protected by copyright. All rights reserved. 


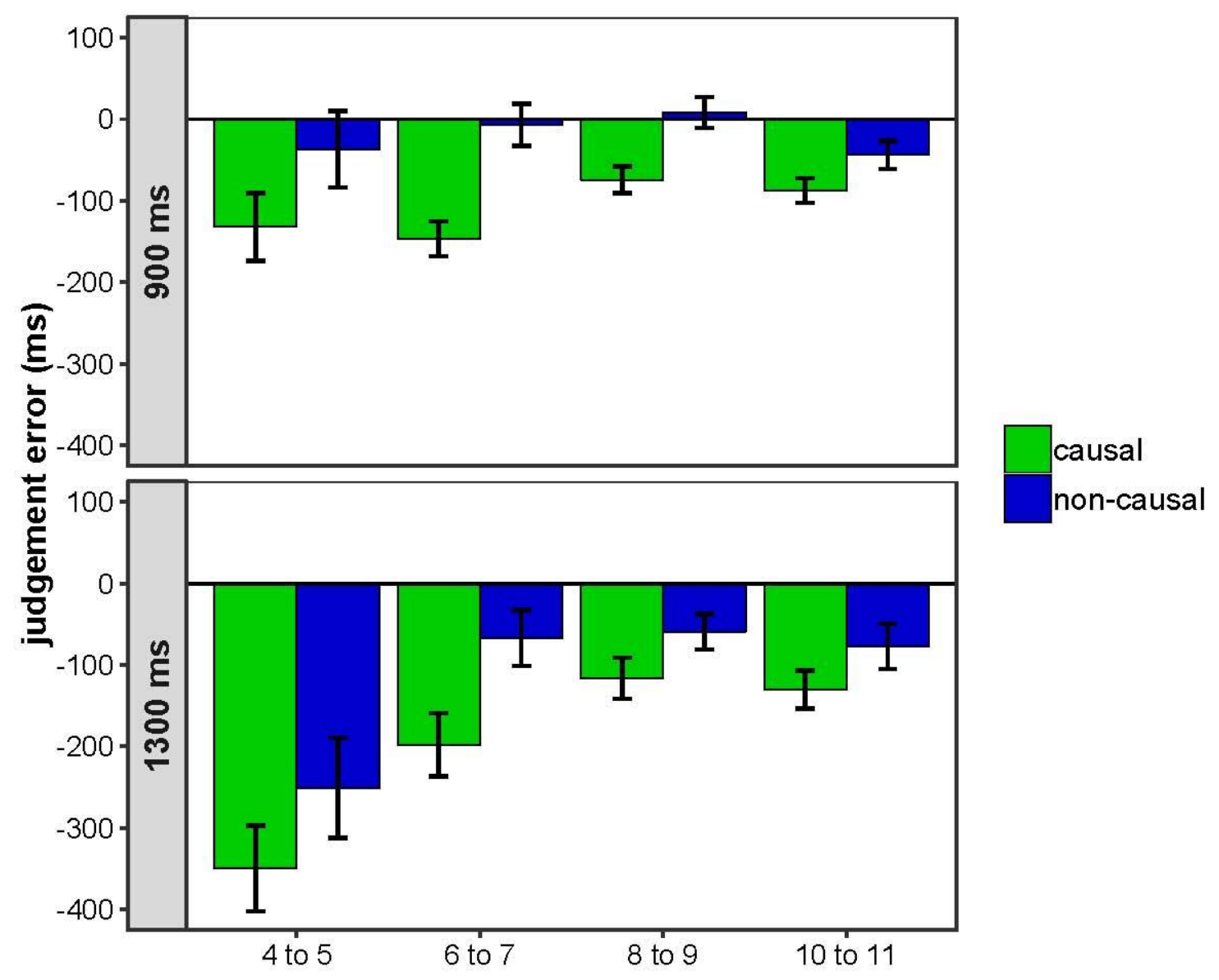

This article is protected by copyright. All rights reserved. 
Figure 4

(a) Experiment 1

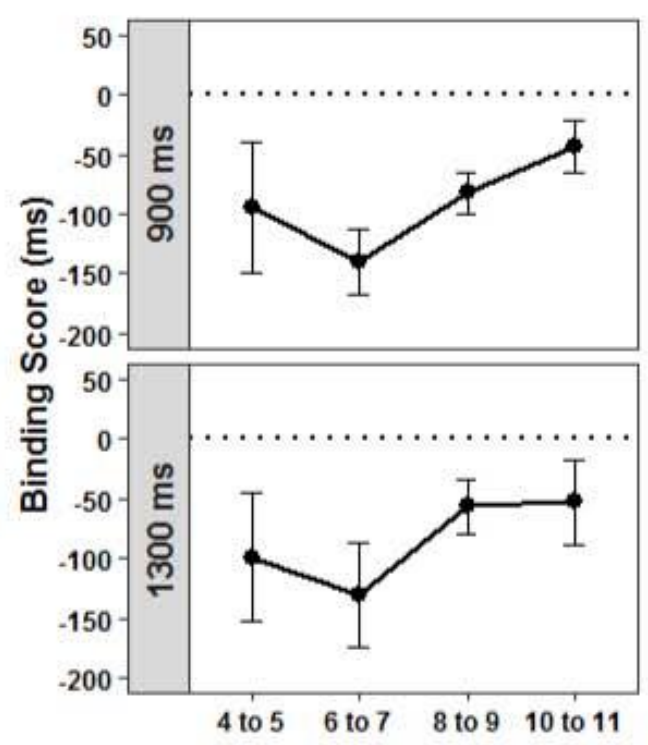

(b) Experiment 2

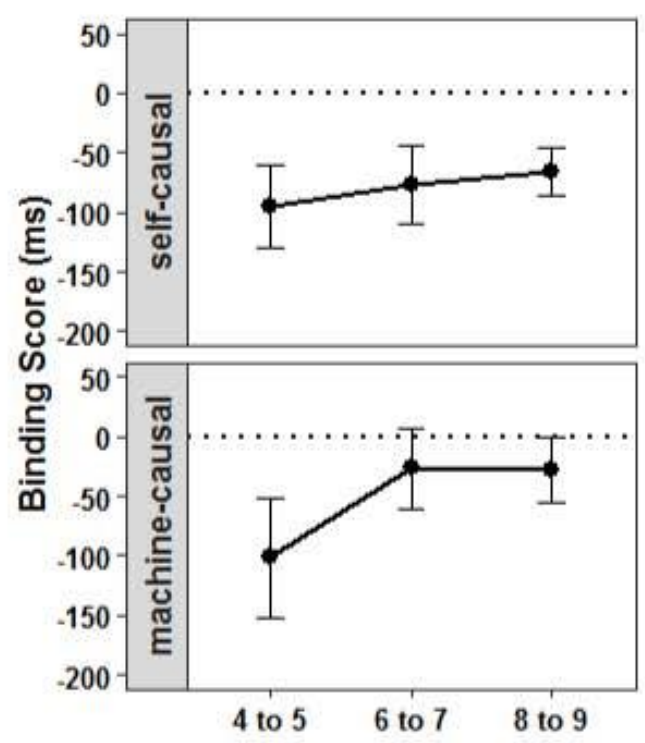

This article is protected by copyright. All rights reserved. 


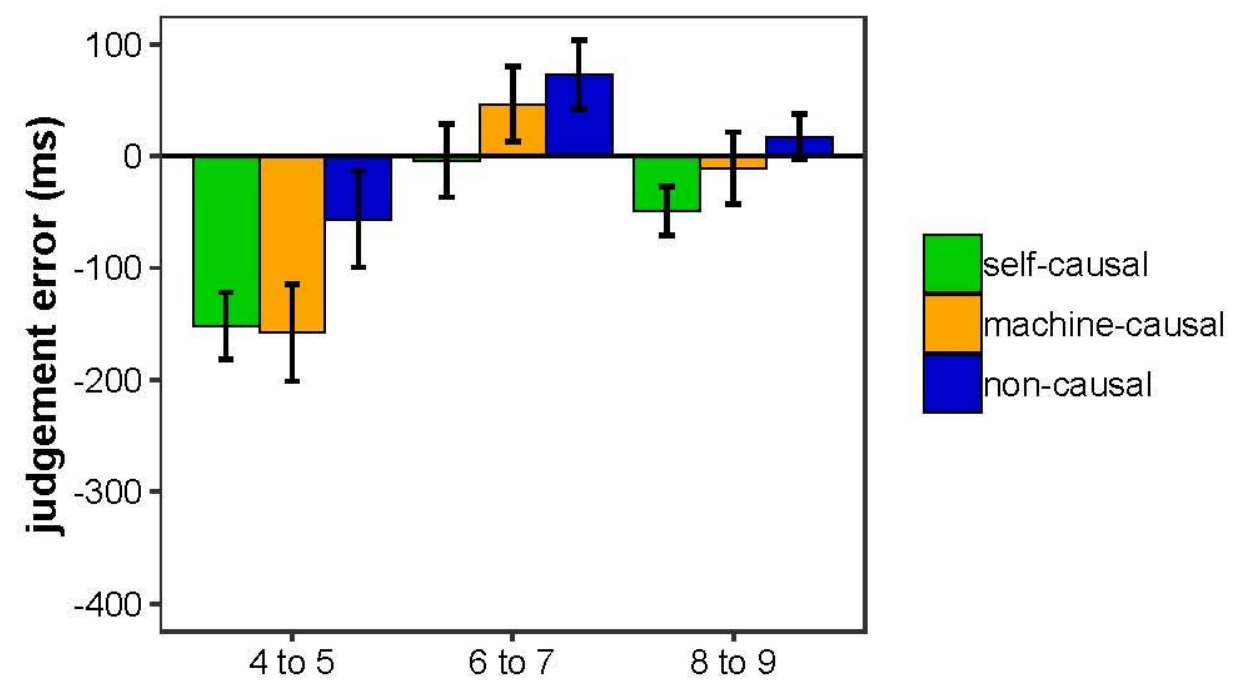

This article is protected by copyright. All rights reserved. 